\title{
Using Auger, EDS, and FIB to Detect, Identify, and Image Buried Metallic Particles
}

Ashley Ellsworth and Dennis Paul

Physical Electronics, Chanhassen, Minnesota, United States

Auger Electron Spectroscopy (AES) is a powerful analytical tool that provides quantitative elemental information from surfaces of solid materials. The average depth of analysis for an AES measurement is approximately $5 \mathrm{~nm}$ with lateral spatial resolution as small as $8 \mathrm{~nm}$. The information AES provides about surface layers or thin film structures is important for many industrial and research applications where surface or thin film composition plays a critical role in performance including: nanomaterials, photovoltaics, catalysis, corrosion, adhesion, semiconductor devices and packaging, magnetic media, display technology, and thin film coatings used for numerous applications.1

In this presentation, defect sites present on a $\mathrm{Ti}$ passivated $\mathrm{Al}$ sheet sample were identified and analyzed using a PHI 710 scanning Auger nanoprobe equipped with a $25 \mathrm{kV}$ Schottky field emission electron gun and a coaxial Cylindrical Mirror Analyzer (CMA). We demonstrate the use of AES in conjunction with a focused ion beam (FIB) to produce site specific imaging of microscale features beneath a sample surface. The combination of the two techniques allows for high spatial resolution analysis of buried particles and defects. This information can be very useful in helping to determine important parameters such as the origin of defects, corrosion mechanisms, coating problems, etc. We will highlight the advantages of FIB milling compared to traditional depth profiling. Energy dispersive $\mathrm{x}$-ray spectroscopy (EDS) is also a powerful complementary technique to Auger analysis as it provides information from much deeper in the sample surface (few $\mu \mathrm{m}$ ). With the combination of AES, FIB, and EDS, we show that buried metal particles can be first located and characterized with EDS, followed by the subsequent FIB milling and Auger spectroscopy of the particles. Auger's surface sensitivity and superior spatial resolution were then used to characterize the true composition and morphology of the freshly exposed particle.

References

1. A. Joshi, D. F. Paul. 2019. ASM Handbook, Volume 10, Materials Characterization. Auger Electron Spectroscopy. ASM Handbook Committee. 